\title{
Efeito da inclusão da covariância genética aditiva direta-materna no modelo de análise sobre a magnitude das estimativas de parâmetros e valores genéticos preditos para ganho de peso na raça Brangus
}

\author{
Effect of genetic direct-maternal covariance inclusion in the model of analizys on the estimate of \\ parameters and predicted genetic values for weight gain in Brangus breed
}

\author{
Luiz Felipe Waihrich Guterres ${ }^{\mathrm{I}}$ Paulo Roberto Nogara Rorato ${ }^{\mathrm{II}}$ Arione Augusti Boligon ${ }^{\text {III }}$ \\ Tomás Weber ${ }^{\text {III }}$ Carlos Junior Kippert ${ }^{\mathrm{IV}}$ Jader Silva Lopes ${ }^{\text {III }}$ \\ Paulo Rodrigo Santos de Souza ${ }^{\mathrm{IV}}$
}

\section{RESUMO}

O objetivo deste trabalho foi estudar o efeito da inclusão da covariância entre o efeito genético aditivo direto e o materno (covd-m) no modelo de análise, sobre o valor das estimativas de parâmetros genéticos e de valores genéticos preditos (VG) para ganho médio diário do nascimento à desmama (GMDND) e da desmama ao sobreano (GMDDS), na raça Brangus (5/8 Angus x 3/8 Nelore). Foram analizados 28.949 registros de desempenho para GMDND e 11.884 para GMDDS, coletados no período entre 1986 e 2002. Os componentes de (co)variância foram obtidos pelo método REML. Para GMDND, foi utilizado um modelo animal que considerou como aleatórios o efeito, genético aditivo direto, o materno e o residual, e como fixos os efeitos de grupo de contemporâneos à desmama (GC205) e da interação fração gênica Nelore-Angus do touro e da vaca (FGNA), além das covariáveis idade da vaca ao parto (ID) e idade do bezerro à desmama. Para GMDDS, o modelo foi o mesmo, apenas substituindo GC205 por grupo de contemporâneos ao sobreano (GC550) e ID por idade ao sobreano. O ambiente permanente da vaca foi considerado como efeito aleatório em ambos os modelos. O teste da razão de verossimilhança mostrou não haver diferença significativa, em nível de 5\% de probabilidade, entre os modelos adotados para ambas as características. As herdabilidades diretas variaram de 0,14 $\pm 0,03$ a 0,21 $\pm 0,03$ $e$ as maternas de 0,00 $\pm 0,01$ a 0,15 \pm 0,02, sendo o valor das estimativas menor quando a covd-m foi considerada no modelo, para GMDND. As correlações entre o valor genético aditivo direto e o materno foram negativas tanto para GMDND (-0,25 $\pm 0,12)$ quanto para GMDDS $(-0,77 \pm 0,19)$. A correlação de ordem ("rank correlation"), entre a classificação dos VG dos animais, preditas pelos dois modelos, foram 0,89 para GMDND e 0,98 para GMDND, sugerindo que pode ocorrer, alteração, embora pequena, na ordem de classificação dos animais em relação a GMDND.

\begin{abstract}
Palavras-chave: efeito de ambiente permanente, herdabilidade, correlação, efeito materno, ganho médio diário do nascimento à desmama, ganho médio diário da desmama ao sobreano.
\end{abstract}

\section{ABSTRACT}

The objective of this research was to study the effect of accouting for the covariance between the additive genetic direct and the maternal effects (covd-m) on the estimates of genetic parameters and on predictions of genetic values (VG), for average daily gain from birth to weaning (GMDND) and from weaning to 550 days of age (GMDDS). They were analyzed 28,949 records for GMDND and 11,884 for GMDDS of a Brangus breed population (518 Angus x 3/8 Nellore), collected from 1986 to 2002. The (co)variance components were obtained by REML. In the animal model for GMDND, the additive genetic direct and maternal and residual effects were considered as random, and the effects of contemporaneous group at weaning (GC205), the interaction of the Nellore-Angus breed genetic percentage of the bull and cow (FGNA) and the covariables, age of the cow at birth (IV) and age at weaning (ID) as fixed effects. For GMDDS, the model was the same, except that GC205 was substituted by contemporaneous group at 550 days of age (CG550) and ID by age at 550 days. In both models, permanent environmental effect of the cow was considered as a random effect. The heritabilities estimated for direct genetic effects ranged from $0.14 \pm 0.03$ to $0.21 \pm 0.03$ and for maternal effects from $0.00 \pm 0.01$ to $0.15 \pm 0.02$, the estimates had smaller values when covd- $m$ was included in the model for GMDND. The correlations between genetic direct and maternal effects were negative $-0.25 \pm 0.12$ (GMDND) and $-0.77 \pm 0.19$ (GMDDS). The likelihood ratio test showed that there is no significant diference, at 5\% significance level, between the adopted models for boths characteristics. The rank correlation

IPlátano-Rastreabilidade e Gestão Agropecuária - Santa Maria, RS, Brasil. E-mail: felipewg@gmail.com.

IIDepartamento de Zootecnia, Universidade Federal de Santa Maria (UFSM), 97105-900, Santa Maria, RS, Brasil. E-mail: rorato@smail.ufsm.br. Autor para correspondência.

IIICurso de graduação em Zootecnia, UFSM, Santa Maria, RS, Brasil.

IVPrograma de Pós-graduação em Zootecnia, UFSM, Santa Maria, RS, Brasil. 
between the VG predicted by the two models, were 0.89 for GMDND and 0.98 for GMDND, suggesting that a slight change in the rank of the animals can happen, for GMDND.

Key words: average daily gain from birth to weaning, average daily gain from weaning to 550 days of age, correlation, heritability, maternal effect, permanent environmental effect.

\section{INTRODUÇÃO}

Os programas de melhoramento de bovinos de corte têm como principal objetivo selecionar animais possuidores de potencial genético superior, que possibilitem a otimização dos sistemas de produção e atendam às exigências do mercado. Por outro lado, as características utilizadas na seleção podem ser afetadas não somente pelo genótipo do animal (efeito direto), como também pelo efeito da mãe (efeito materno), particularmente no período pré-desmama. MARCONDES et al. (2000) relataram que a seleção de animais com o objetivo de obter altos ganhos de peso na pré-desmama favorece a obtenção de animais que ficam prontos, com o peso exigido pelo mercado, mais rapidamente. Portanto, conhecer a influência materna sobre os pesos pré e pós-desmama e a correlação entre o efeito genético aditivo direto e o materno é de fundamental importância para a obtenção de estimativas de herdabilidade precisas e não viciadas (CABRERA et al., 2001).

A influência do efeito materno sobre o ganho de peso, na raça Nelore, na Região Nordeste, foi relatada por BIFFANI et al. (1999). Por outro lado, CARDOSO et al. (2004) afirmaram que a inclusão do efeito materno no modelo de análise tendeu a influenciar a estimativa da variância genética aditiva, dependendo da correlação entre o efeito genético direto e o materno, na raça Angus.

Diferentemente ao proposto por LÔBO et al. (2000), de considerar a covariância entre o efeito genético aditivo direto e o materno igual a zero, são encontrados na literatura trabalhos estudando o efeito da inclusão desta covariância no modelo de análise sobre as estimativas de parâmetros genéticos, bem como sobre o valor genético predito (CABRERA et al., 2001; MALHADO et al., 2003; MALHADO et al., 2004), não havendo consenso entre os resultados.

O objetivo do presente trabalho foi estudar a possível influência da inclusão da covariância entre o efeito genético aditivo direto e o materno, nos modelos de análise, sobre os valores das estimativas dos parâmetros genéticos para ganho médio diário do nascimento à desmama e da desmama ao sobreano e sobre o ordenamento (rank) dos animais pelos seus valores genéticos preditos.

\section{MATERIAL E MÉTODOS}

Foram analisados 28.949 e 11.884 registros de desempenho de bovinos da raça Brangus (5/8 Angus x 3/8 Nelore), para avaliar o efeito da inclusão da covariância entre o efeito genético aditivo direto e o materno (covd-m) no modelo, sobre as estimativas de parâmetros genéticos e das predições dos valores genéticos dos animais, para as características ganho médio diário do nascimento à desmama (GMDND) e ganho médio diário da desmama ao sobreano (GMDDS), respectivamente. Os dados foram coletados em fazendas de diferentes regiões do Brasil, no período entre 1986 e 2002, e foram cedidos por Gensys Consultores Associados S/C Ltda. e Natura Genética Sul-Americana.

Na montagem do arquivo de trabalho, foram eliminados os registros de animais com GMDND inferiores a $440,70 \mathrm{~g}$ ou superiores a $955,70 \mathrm{~g}$ e com GMDDS inferiores a $141,60 \mathrm{~g}$ ou superiores a $620,50 \mathrm{~g}$. Os limites para exclusão dos dados foram obtidos considerando 2,5 desvios-padrão, para mais ou para menos, em relação à média. Foram geradas as variáveis grupo de contemporâneos ao nascimento-GCN, à desmama-GC205 e ao sobreano-GC550 (animais nascidos, desmamados e pesados ao sobreano, respectivamente, na mesma fazenda, ano e estação e pertencentes ao mesmo sexo); estação de nascimento, de desmama e de sobreanao: (1) animais nascidos nos meses de janeiro a março, (2) de abril a junho, (3) de julho a setembro e (4) de outubro a dezembro; interação da fração gênica Nelore-Angus touro-vaca (FGNA), conforme tabela 1 .

Os componentes de (co)variância foram obtidos pelo programa computacional MTDFREML, descrito por BOLDMAN et al. (2001). Para GMDND, foi adotado um modelo animal que considerou como aleatórios os efeitos genéticos aditivos diretos e maternos e o residual, e como fixos os efeitos de GC205, das covariáveis idade do bezerro a desmama (ID) e idade da vaca ao parto (efeitos linear e quadrático). Para GMDDS, o modelo foi o mesmo, apenas substituindo-se GC205 por GC550 e ID por idade ao sobreano (IS). O efeito aleatório de ambiente permanente da vaca foi incluído nos modelos para as duas características. Cada modelo foi utilizado em duas análises, uma considerando a covd-m igual a zero e a outra considerando um valor previamente estimado. Os modelos de análise utilizados podem ser descritos, sob a forma matricial, como: $\mathrm{Y}=\mathrm{X} \beta+\mathrm{Z}_{1} \mathrm{a}+\mathrm{Z}_{2} \mathrm{~m}+\mathrm{Z}_{3} \mathrm{p}+\mathrm{e}$, onde: $\mathrm{Y}$ = vetor das observações de cada característica (GMDND e GMDDS); $\mathrm{X}=$ matriz de incidência associada aos efeitos fixos; $\beta$ = vetor de solução para 
Tabela 1 - Distribuição das freqüências de acordo com os grupos genéticos Nelore-Angus dos pais para GMDND e GMDDS.

\begin{tabular}{|c|c|c|c|c|}
\hline \multirow{2}{*}{ Grupos Genéticos Nelore-Angus dos Pais } & \multicolumn{2}{|c|}{ Freqüência } & \multicolumn{2}{|c|}{$\%$} \\
\hline & GMDND & GMDDS & GMDND & GMDDS \\
\hline $0,0000-0,7960$ & 4.754 & 1343 & 16,42 & 11,30 \\
\hline $0,3750-0,3750$ & 17.746 & 7434 & 61,30 & 62,55 \\
\hline $0,3750-0,5000$ & 287 & 28 & 0,99 & 0,24 \\
\hline $0,5000-0.3750$ & 3 & - & 0,01 & - \\
\hline $1,0000-0,0000$ & 6.159 & 3079 & 21,28 & 25,91 \\
\hline
\end{tabular}

os efeitos fixos; $Z_{1}=$ matriz de incidência associada ao efeito genético aditivo direto de cada animal; $\mathrm{a}$ = vetor de soluções para os efeitos genéticos aditivos diretos aleatórios; $\mathrm{Z}_{2}=$ matriz de incidência associada ao efeito genético aditivo materno de cada animal; $\mathrm{m}$ = vetor de soluções para os efeitos genéticos aditivos maternos aleatórios; $\mathrm{Z}_{3}=$ matriz de incidência associada ao efeito de ambiente permanente da vaca; $p$ = vetor de soluções para os efeitos aleatórios de ambiente permanente da vaca; e = vetor dos resíduos

O critério de convergência considerado foi $10^{-6}$, sendo que, a cada convergência, o programa era reiniciado, utilizando-se como valores iniciais as estimativas da análise anterior, até o momento em que o resultado do $-2 \log _{e}$ da função de verossimilhança não se alterava mais nas últimas três análises; então, o procedimento era finalizado. A matriz de parentesco estava constituída de 47.489 animais para o arquivo de GMDND e 20.571 animais para o arquivo de GMDDS.

Os modelos foram comparados pelo teste de razão de verossimilhança ( 1 ) com probabilidade de erro de 5\%. Segundo FREUND \& WALPOLE (1980), lé calculada como segue: l = L2/L1, em que: $\mathrm{L} 2$ = valor de máxima verossimilhança do modelo 2 (com a inclusão da covariância aditiva direta-materna); $\mathrm{L} 1$ = valor de máxima verossimilhança do modelo 1 (sem a inclusão da covariância aditiva direta-materna). Ainda, segundo esses autores, para um grande número de observações (n), a distribuição de -2logl aproxima-se, via de regra, a uma distribuição de qui-quadrado (c2) com 1 grau de liberdade, i.e.: $-2 \log l \sim \mathrm{c}^{2} \mathrm{an}$, onde $\mathrm{a}=$ nível de significância e n = grau de liberdade (1). O cálculo de 2logl pode ser simplificado aplicando-se a diferença entre -2logL2 e -2logL1, uma vez que: $\mathrm{I}=\mathrm{L} 2 / \mathrm{L} 1$ = L2.L1$1, \mathrm{p} \log \mathrm{l}=\log \mathrm{L} 2-\log \mathrm{L} 1, \mathrm{P}-2 \log \mathrm{l}=(-2 \log \mathrm{L} 2)-(-$ $2 \log L 1)$. O valor obtido para a estatística do teste da razão de verossimilhança (l) foi comparado com o valor do qui-quadrado tabelado (c2tab) com um grau de liberdade, considerando $l>c^{2}$ tab $=$ efeito significativo.

Todos os animais com registros de desempenho no arquivo de trabalho foram ordenados pelos seus valores genéticos preditos nas duas análises (com e sem a inclusão da covariância direto-materna) e a correlação entre os ordenamentos ("rank correlation”) foi estimada pelo procedimento CORR, opção Spearman, do programa SAS (2001), com o objetivo de avaliar a correspondência entre as classificações dos reprodutores obtidas pelos diferentes modelos de análise.

\section{RESULTADOS E DISCUSSÃO}

A média observada (Tabela 2), em gramas, para GMDND $(699,29)$ foi cerca de $13 \%$ superior àquela relatada por CARDOSO et al., 2004 (605g), para a raça Angus e inferior à verificada por CABRERA et al. (2001) (787g), para a raça Nelore. Para GMDDS, a média observada foi 346,39g, próxima à encontrada por CARDOSO et al. (2004) (352g), para a raça Angus e superior à relatada por CABRERA et al. (2001) (295g), para a raça Nelore. O coeficiente de variação para GMDDS, fase pós-desmama, foi duas vezes maior do que aquele encontrado para GMDND, fase prédesmama, em conseqüência da maior variabilidade observada nos ganhos diários nesta última fase.

As estimativas obtidas para os componentes de variância genética aditiva e de ambiente permanente para GMDND (Tabela 3) foram maiores e a variância residual decresceu, quando a covd-m foi considerada no modelo, concordando com CABRERA et al. (2001), MALHADO et al. (2003) e MALHADO et al. (2004). Entretanto, diferentemente do relatado por esses autores, neste trabalho, a variância do efeito materno decresceu quando a covd-m foi considerada no modelo. Para GMDND, a inclusão da covd-m no modelo promoveu aumento na variância genética aditiva direta e redução na variância residual, concordando com MALHADO et al. (2003). A redução na variância residual sugere um melhor ajuste nos dados quando a covd-m é incluída no modelo.

A herdabilidade direta para GMDND foi cerca de $11 \%$ maior quando a covd-m foi considerada, concordando com CABRERA et al. (2001), segundo os 
Tabela 2 - Número de observações (N), médias, coeficientes de variação (CV\%), valores mínimo (Min.) e máximo (Máx.), para ganho médio diário de peso do nascimento à desmama (GMDND) e da desmama ao sobreano (GMDDS), idade da vaca ao parto (ID VACA), idade ao desmame (ID DESM), peso ao desmame (P205) e peso ao sobreano (P550).

\begin{tabular}{|c|c|c|c|c|c|}
\hline Característica & $\mathrm{N}$ & Média & $\mathrm{CV}$ & Min. & Máx. \\
\hline \multicolumn{6}{|c|}{ Desmama } \\
\hline GMDND (g) & 28.949 & 699,29 & 16,37 & 440,70 & 955,70 \\
\hline ID VACA (dias) & 28.949 & 2.022 .49 & 39,93 & 382,00 & $6.995,00$ \\
\hline ID DESM (dias) & 28.949 & 208,41 & 16,34 & 90,00 & 328,00 \\
\hline P205 (kg) & 28.949 & 173,35 & 13,63 & 119,00 & 227,00 \\
\hline \multicolumn{6}{|c|}{ Sobreano } \\
\hline GMDDS (g) & 11.884 & 346,39 & 33,37 & 141,60 & 620,50 \\
\hline ID VACA (dias) & 11.884 & $2.050,82$ & 40,13 & 382,00 & $6.654,00$ \\
\hline ID SOB (dias) & 11.884 & 206,66 & 13,40 & 309,60 & 801,80 \\
\hline P550 (kg) & 11.884 & 292,95 & 16,91 & 164,40 & 451,30 \\
\hline
\end{tabular}

quais as herdabilidades também aumentaram, discordando assim de MALHADO et al. (2003), que relataram o mesmo valor de herdabilidade $(0,11)$ com a inclusão ou não da covd-m no modelo. A herdabilidade materna decresceu cerca de $13 \%$ quando a covd-m foi considerada, discordando de CABRERA et al. (2001) e de MALHADO et al. (2003), os quais verificaram aumento no valor desta estimativa quando covd-m foi considerada no modelo. As herdabilidades diretas estimadas para GMDND foram similares à relatada por MARCONDES et al., $2002(0,19)$, e superiores às relatadas por MARCONDES et al. (2000) $(0,11$ a 0,20), MALHADO et al., 2003 (0,12), SARMENTO et al., 2003 $(0,12)$ e MALHADO et al., 2004 (0,12); as herdabilidades maternas foram similares às estimadas por MARCONDES et al., $2000(0,03$ a 0,16), e superiores às relatadas por MARCONDES et al., $2002(0,10)$, MALHADO et al., $2003(0,05$ a 0,06) e MALHADO et al., 2004 (0,07 a 0,09).

Em relação a GMDDS, a inclusão da covdm no modelo promoveu aumento de cerca de $21 \%$ no valor da herdabilidade direta, concordando com CABRERA et al. (2001) e discordando de MALHADO et al. (2003) para os quais a inclusão da covd-m não alterou o valor da estimativa. Para GMDDS, as herdabilidades diretas foram similares à relatada por MARCONDES et al., 2000, (0,18); inferiores à relatada por MARCONDES et al., $2002(0,49)$ e SARMENTO et al., 2003, (0,21), e superiores à estimativa de MALHADO et al., 2003, (0,14).

As correlações genéticas (Tabela 3) entre os efeitos direto e materno foram negativas, discordando de MERCADANTE \& LÔBO (1997) e concordando com MEYER (1994), CARDOSO et al. (2001), EVERLING et al. (2001), MALHADO et al.(2003) e SARMENTO et al. (2003). Este fato, segundo ELER et al. (1989), pode comprometer uma resposta positiva à seleção, para potencial de crescimento do bezerro, em decorrência do efeito materno. Quanto à magnitude das estimativas, os resultados deste trabalho são inferiores aos relatados por CARDOSO et al. (2001), EVERLING et al. (2001) e SARMENTO et al. (2003), e superiores ao relatado por MALHADO et al. (2003), para GMDND. Estes valores negativos de correlação, variando de médio a alto, sugerem um antagonismo entre os efeitos dos genes relacionados ao potencial de crescimento do terneiro e à habilidade materna, sendo esta, neste período, a maior fonte de influência sobre o desenvolvimento do terneiro. Resultados negativos de correlações entre o efeito genético aditivo direto e o materno são comumente encontrados na bibliografia, também para outras características de crescimento, como peso ao nascer, peso à desmama e ao sobreano. Autores como ELER et al. (1995); MAGNABOSCO et al. (1996); MEYER (1997); CABRERA et al. (2001); CARDOSO et al. (2001) e RIBEIRO et al. (2001) afirmam que os valores negativos encontrados para esta correlação são devidos mais à inadequação dos dados a metodologias e modelos adotados para as análises do que, propriamente, a causas biológicas. Segundo ELER et al. (2000), uma outra provável explicação para a ocorrência de correlações negativas seria o fato da não inclusão do efeito da interação touro x rebanho nos modelos de análise. Esta evidência surgiu após resultados de trabalhos com correlações próximas a zero e positivas, em estudos onde a interação tourorebanho foi incluída.

A correlação de ordem entre a classificação dos animais pelo valor genético predito foi 0,98 para GMDDS, mostrando uma correspondência próxima de $100 \%$ nas estimativas obtidas pelos dois modelos; entretanto, para GMDND, esta correlação foi 0,89 , 
Tabela 3 - Componentes de (co) variância e parâmetros genéticos estimados, para ganho médio diário do nascimento a desmama (GMDND) e da desmama ao sobreano (GMDDS).

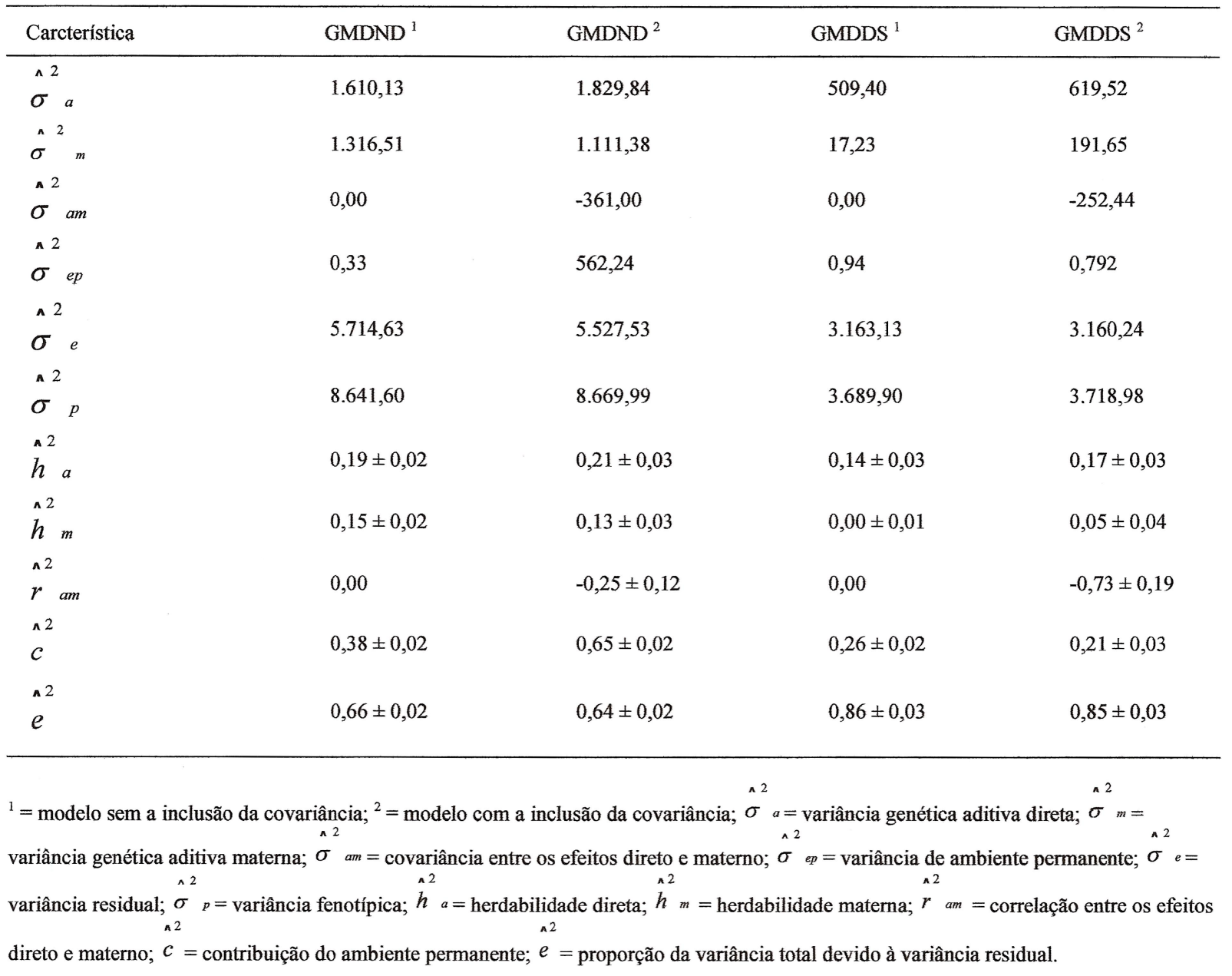

sugerindo alguma alteração no VG e, por conseqüência, na classificação dos animais. Este fato pode induzir a erros na identificação dos animais superiores. Segundo MALHADO et al. (2004), à medida que diminui a proporção de animais selecionados, aumenta a diferença na classificação, e concluindo que a covariância direta-materna pode influenciar bastante a seleção de animais superiores, merecendo, por isso, mais atenção e estudos.

Apesar de o teste de razão de verossimilhança ter mostrado não haver diferença significativa entre os modelos, em nível de $5 \%$ de probabilidade, para ambas as características, as estimativas de herdabilidade diferentes, para maior quando a covariância genética-materna foi considerada no modelo (11\% para GMDND e 21\% para GMDDS), sob o ponto de vista prático, podem induzir a erros nos procedimentos seletivos, no que se refere à resposta esperada da seleção.

\section{CONCLUSÕES}

Ao se estimar parâmetros genéticos para a característica ganho médio diário de peso do nascimento à desmama, a covariância entre o efeito genético aditivo direto e o materno deve ser considerada no modelo, uma vez que sua inclusão promove redução no componente de variância residual, conferindo melhor ajuste aos dados. Entretanto, para a característica ganho médio de peso da desmama ao sobreano isso não é necessário.

Deve-se, portanto, proceder a inclusão da covariância entre os efeitos genéticos aditivos diretos e maternos no modelo de análise para estimar o valor genético de reprodutores, para a característica ganho de peso médio diário do nascimento à desmama, uma vez que a sua não-inclusão pode provocar alteração na ordem de classificação dos animais. 


\section{AGRADECIMENTOS}

Os autores agradecem a Gensys Consultores Associados S/C Ltda. e a Natura Genética Sul-Americana, pela cedência dos arquivos de dados, e ao CNPq, pela concessão de bolsa PIBIC.

\section{APRESENTAÇÃo}

Parte da dissertação apresentada pelo primeiro autor ao Programa de Pós-graduação em Zootecnia da Universidade Federal de Santa Maria, como parte dos requisitos para a obtenção do grau de Mestre em Produção Animal.

\section{REFERÊNCIAS}

BIFFANI, S. et al. Fatores ambientais e genéticos sobre o crescimento ao ano e ao sobreano de bovinos Nelore, criados no Nordeste do Brasil. Revista Brasileira Zootecnia, v.28, n.4, p.468-473, 1999.

BOLDMAN, K.G. et al. A manual for use of MTDFREML. A set of programs to obtain estimates of variances and covariances. (Draft). Lincoln: Department of Agriculture, Agricultural Research Service, 2001. 117p.

CABRERA, M.E. et al. Efecto de la incorporación de la covarianza genética directa-materna en el análisis de características de crescimiento em la raza Nelore. Livestock Research for Rural Development, v.13, n.3, p.1-6, 2001.

CARDOSO, F.F. et al. Componentes de (co)variância e parâmetros genéticos para caracteres produtivos à desmama de bezerros Angus criados no estado do Rio Grande do Sul. Revista Brasileira Zootecnia, v.30, n.1, p.41-48, 2001.

CARDoso, F.F. et al. Componentes de (co)variância e parâmetros genéticos para caracteres pós-desmama e bovinos da raça Angus. Revista Brasileira Zootecnia, v.33, n.2, p.313-319, 2004.

ELER, J.P. et al. Avaliação dos efeitos genéticos direto e materno em pesos de bovinos da raça Nelore criados no estado de São Paulo. Revista Brasileira Zootecnia, v.18, n.2, p.112-123, 1989.

ELER, J.P. et al. Estimation of variances due to direct and maternal effects for growth traits of Nelore cattle. Journal of Animal Science, v.73, p.3253-3258, 1995.

ELER, J.P. et al. Influência da interação touro-rebanho na estimação da correlação entre efeitos genéticos direto e materno em bovinos da raça Nelore. Revista Brasileira Zootecnia, v.29, n.6, p.1642-1648, 2000.

EVERLING, D.M. et al. Estimativas de herdabilidade e correlação genética para características de crescimento na fase de prédesmama e medidas de perímetro escrotal ao sobreano em bovinos Angus-Nelore. Revista Brasileira Zootecnia, v.30, n.6, p.2002-2008, 2001. (suplemento 1).
FREUND, J.F.E.; WALPOLE, R.E. Mathematical Statistics. 3.Ed. New Jersey: Prentice-Hall, 1980. 548p.

LÔBO, R.N.B et al. Getic parameters for growth traits of zebu cattle in the semi-arid region of Brazil. Ciência Animal, v.10, n.1, p.7-12, 2000.

MAGNABOSCO, C.U. et al. Estimativas de parâmetros genéticos e de ambiente de características de crescimento em bovinos da raça Nelore. In: REUNIÃO ANUAL DA SOCIEDADE BRASILEIRA DE ZOOTECNIA, 33., 1996, Fortaleza, CE. Anais... Fortaleza: Sociedade Brasileira de Zootecnia, 1996. v.1, p.142-144.

MALHADO, C.H. et al. Efeito da inclusão da covariância entre os efeitos direto e materno sobre os parâmetros genéticos de ganho de peso pré-desmama e pós-desmama. In: REUNIÃO ANUAL DA SOCIEDADE BRASILEIRA DE ZOOTECNIA, 40., 2003, Santa Maria, RS. Anais... Santa Maria: Sociedade Brasileira de Zootecnia, 2003. CD-Rom

MALHADO, C.H. et al. Efeito da incorporação da covariância entre os efeitos direto e materno sobre a análise para a característica dias para ganhar $160 \mathrm{Kg}$. Brazilian Journal of Veterinary Research and Animal Science, v.41, n.1, p.1419, 2004.

MARCONDES, C.R. et al. Análise de alguns critérios de seleção para características de crescimento na raça Nelore. Arquivo Brasileiro de Medicina Veterinária e Zootecnia, v.52, n.1, p.83-89, 2000.

MARCONDES, C.R. et al. Análise de alguns critérios de seleção para características de crescimento na raça Nelore. Arquivo Brasileiro de Medicina Veterinária e Zootecnia, v.54, n.1, p.93-99, 2002.

MERCADANTE, M.E.Z.; LÔBO, R.B. Estimativas de (co)variância e parâmetros genéticos e de ambiente de característica de crescimento de fêmeas de um rebanho Nelore. Revista Brasileira Zootecnia, v.26, n.6, p.1124-1133, 1997.

MEYER, K. Estimates of direct and maternal correlations among growth traits in Australian beef cattle. Livestock Production Science, v.38, p.91-105, 1994.

MEYER, K. Estimates of genetic parameters for weaning weight of beef cattle accounting for direct-maternal enviromental covariances. Livestock Production Science, v.52, p.187199, 1997.

RIBEIRO, M.N. et al. Herdabilidade para efeitos direto e materno de características de crescimento de bovinos Nelore no estado da Paraíba. Revista Brasileira Zootecnia, v.30, n.4, p.1224-1227, 2001.

SARMENTO, J.L.R. et al. Efeitos ambientais e genéticos sobre o ganho de peso diário de bovinos Nelore no estado da Paraíba. Revista Brasileira Zootecnia, v.32, n.2, p.325-330, 2003.

SAS. Statistical Analysis Systems user's guide: Stat, Version 8.ed. Cary, 2001. 956p. 